\title{
Evaluation of Different Pretreatment Efficacy with Fluoride-releasing Material on Shear Bond Strength of Orthodontic Bracket: An In Vitro Study
}

\author{
Nitin R Khargekar ${ }^{1}$, Jubin H Kalathingal ${ }^{2}$, George Sam ${ }^{3}$, Mohamed A Elpatal ${ }^{4}$, Sadananda Hota ${ }^{5}$, Purnendu Bhushan ${ }^{6}$
}

\begin{abstract}
Aim: The aim of this study was to assess the efficacy of different pretreatments with fluoride-releasing material on shear bond strength (SBS) of orthodontic bracket.

Materials and methods: A total of 60 human mandibular premolars were taken in this study. These samples were stored in periodically changed distilled water at room temperature so as to stop bacterial growth. All samples were randomized to three pretreatment groups: group I, acid etching; group II, fluoride varnish; group III, casein phosphopeptide-amorphous calcium phosphate (CPP-ACP) paste. Later, Transbond XT was applied on brackets made of stainless steel and brackets were centered buccally and then light-cured. The INSTRON universal machine was used to measure SBS. Based on the values, the adhesive remnant index (ARI) was estimated.

Results: The maximum SBS was seen in group III (18.84 \pm 1.04$)$, followed by group II (14.18 \pm 0.23$)$, and the minimum bond strength was found in group I ( $13.90 \pm 1.22)$. The one-way analysis of variance (ANOVA) showed high statistically significant difference within the pretreatment groups. The pretreatment group comparisons showed statistically significant difference between group I vs group III and group II vs group III. Score 0 was more in group III [8 (40\%)] followed by group II [4 (20\%)] and group I [2 (10\%)]. The lowest number of score 3 was found in CPP-ACP paste group. After comparing the ARI scores within the groups, the Chi-square test showed a probability of 0.001 which was statistically significant. Conclusion: Our study established that the CPP-ACP paste pretreatment improves the SBS of orthodontic bracket significantly followed by fluoride varnish and acid etching.

Clinical significance: The bond strength of orthodontic brackets that were bonded should be adequate to resist orthodontic forces applied during treatment. The effect of the adhesive material to improve the adequate bond strength depends on the efficient pretreatment methods. Keywords: Adhesive bond strength, Orthodontic bracket, Pretreatment, Universal testing machine.

The Journal of Contemporary Dental Practice (2019): 10.5005/jp-journals-10024-2697
\end{abstract}

\section{INTRODUCTION}

The maintenance of oral hygiene gets compromised during orthodontic treatment because of the existence of bands, brackets, coils, arch wires, and springs. Additionally, acid-etching procedure results in loss (about 5-10 $\mu \mathrm{m}$ ) of enamel surface which leads to permanent demineralization of enamel surface around or beneath the orthodontic bracket. Also, dental plaque deposits easily in areas around the bracket base wherever there is extra bonding material. Altogether, these conditions finally upsurge the possibility of early caries during orthodontic treatment. ${ }^{1}$

For successful bonding of brackets, the tooth enamel should remain strong and healthy; however, the process of dental caries and erosion commonly causes loss of inorganic/mineral components of tooth. Dental caries comprises loss of mineral components of tooth by chemical dissolution due to a fall in $\mathrm{pH}$ of dental biofilm. Dental erosion encompasses loss of mineral components of tooth by non-bacterial acidic substances which comes in contact with the tooth structure. ${ }^{2}$

Use of fluoride (F) as an intraoral preventive or therapeutic agent has proven to be efficacious and has made a substantial influence on patient's health and quality of life time after time. There have been good results from several trials of fluoride-releasing materials which display the efficiency of fluoride in controlling the progress of caries. Fluoride prevents the caries progression either by stopping demineralization or by initiating remineralization of enamel/dentin. ${ }^{3}$
${ }^{1}$ Department of Orthodontics and Dentofacial Orthopedics, NSVK Sri Venkateshwara Dental College and Hospital, Bengaluru, Karnataka, India

${ }^{2}$ Kings Dental Center, Qatar

${ }^{3}$ Department of Preventive Dental Sciences, College of Dentistry, Prince Sattam Bin Abdul Aziz University, Al-Kharj, Kingdom of Saudi Arabia

${ }^{4}$ Department of Pedodontics and Oral Health, College of Dentistry, Al-Azhar University, Cairo, Egypt

5,6Department of Prosthodontics, Kalinga Institute of Dental Sciences, KIIT Deemed to be University, Bhubaneswar, Odisha, India

Corresponding Author: Nitin R Khargekar, Department of Orthodontics and Dentofacial Orthopedics, NSVK Sri Venkateshwara Dental College and Hospital, Bengaluru, Karnataka, India, Phone: +91 9448647970,e-mail:nkhargekar@yahoo.co.in

How to cite this article: Khargekar NR, Kalathingal JH, Sam G, et al. Evaluation of Different Pretreatment Efficacy with Fluoride-releasing Material on Shear Bond Strength of Orthodontic Bracket: An In Vitro Study. J Contemp Dent Pract 2019;20(12):1442-1446.

Source of support: Nil

Conflict of interest: None

Ideally, an adhesive agent should possess adequate bond strength to resist excessive forces that affect the bonding of the

() The Author(s). 2019 Open Access This article is distributed under the terms of the Creative Commons Attribution 4.0 International License (https://creativecommons. org/licenses/by-nc/4.0/), which permits unrestricted use, distribution, and non-commercial reproduction in any medium, provided you give appropriate credit to the original author(s) and the source, provide a link to the Creative Commons license, and indicate if changes were made. The Creative Commons Public Domain Dedication waiver (http://creativecommons.org/publicdomain/zero/1.0/) applies to the data made available in this article, unless otherwise stated. 
brackets. Simultaneously, the bonding agent should be able to avoid demineralization of tooth structure. The ability of systemic and topical fluorides to prevent the progression of dental caries has led to the development of various clinical trials to create and sustain low levels of intraoral free fluoride that aids the cariostatic mechanism. ${ }^{4}$

During fixed orthodontic treatment, progression of dental caries can be avoided by the use of adhesive agents that release fluoride. These fluoride-releasing adhesive cements can adhere to base metal alloys and are so suggested for the direct bonding of brackets during orthodontic therapy. ${ }^{5}$ Therefore, the current study was done to assess the different pretreatment efficacy with fluoride-releasing material on shear bond strength (SBS) of orthodontic bracket.

\section{Materials and Methods}

The present in vitro study was conducted in the Department of Orthodontics, Nehru Smaraka Vidya Kendra Sri Venkateshwara Dental College and Hospital, Bengaluru, India.

The trial comprised totally of 60 human mandibular premolar teeth that were extracted for reasons related to orthodontic treatment. Undamaged, healthy, caries-free, teeth with no restorations, or developmental defects buccally were used. The samples were kept in distilled water at room temperature. In order to avoid bacterial growth, the samples were moved to fresh distilled water on a periodic basis. All the samples were fixed on acrylic block such that only the coronal portions of the teeth were visible. All 60 samples (20 samples in each group) were randomly dispersed to three pretreatment groups.

\section{Pretreatment Groups}

\section{Group I: Acid Etching (Control Group)}

For the teeth belonging to this group, acid-etching was performed on the buccal surface for 30 seconds using 35\% phosphoric acid (Ultra Etch, Ultradent Products, Inc.). After etching, the teeth were rinsed with water spray for 15 seconds and dried by air until the teeth were desiccated.

\section{Group II: Fluoride Varnish (Bifluorid 12)}

The buccal enamel surface was completely cleaned and dried. A thin layer of Bifluorid 12 varnish was applied using the viva brush. It was evenly dispersed with an air syringe and after 45 minutes bonding was done.

\section{Group III: CPP-ACP Paste (GC Tooth Mousse Plus)}

A CPP-ACP paste of pea size was applied on the buccal surface of teeth. According to the instructions of the manufacturer, the paste was retained up to half an hour prior the bonding procedure.

The guidelines and instructions by the manufacturer were followed for the bonding procedure. The primer (Transbond ${ }^{\mathrm{TM}} \mathrm{XT}$ primer) from 3M Unitek, USA, was applied on the enamel surface that has been acid-etched. After application of Transbond XT ( $3 \mathrm{M}$, Monrovia, CA, USA) on stainless-steel brackets, the bracket was centered on the buccal surface of the crown and was then lightcured. Every bracket was light-cured for 20 seconds from both distal and mesial sides. Samples were kept in distilled water for 24 hours at $37^{\circ} \mathrm{C}$ and then were thermocycled (Fig. 1) to expose the teeth to the changes in the temperature in the same way as it occurs in the mouth for 1,000 cycles in distilled water at
$5 \pm 2^{\circ} \mathrm{C}-55 \pm 2^{\circ} \mathrm{C}$ with a storage time of 30 seconds and a transfer time of 10 seconds.

\section{Evaluation of Shear Bond Strength}

The samples were placed in a particular position in the INSTRON universal machine's (INSTRON 3365, UK) (Fig. 2) mounting jig so that the bracket base faced the shear-peel load parallelly. The teeth were positioned with a stainless-steel wire measuring $0.019 \times 0.025$ inch to confirm that mounting of all the brackets was in the similar alignment in relation to the acrylic cylinder. This technique for mounting warrants uniformity in the debonding force direction and force application point. A shear debonding force was applied in a gingivo-occlusal direction to the base of the bracket at a crosshead speed of $1 \mathrm{~mm} /$ minute. The highest force required to debond or start fracture of the bracket was measured in Newtons and then changed to megapascals ( $\mathrm{MPa}$ ) as a ratio of Newtons to surface area of bracket base. Random examination of debonded samples at a magnification of $40 \times$ to assess the bond failure site.

\section{Evaluation of Adhesive Remnant Index}

Post debonding and as per Artun and Bergland, ${ }^{6}$ the fractured specimen was examined and ARI was estimated (Fig. 3).

Score 0 -no adhesive remaining on the tooth.

Score 1-less than half of the adhesive remaining on the tooth. Score 2-more than half of the adhesive remaining on the tooth.

Score 3-all adhesive remaining on the tooth, with a prominent impression of the bracket mesh.

\section{Statistical Analysis}

A SPSS 20.0 software (Chicago Inc., USA) was used for statistical analysis. Descriptive statistics (mean, standard deviation, and significance) of bond strength was estimated for all groups. Statistical tests such as ANOVA and Chi-square test were carried out for bond strength and ARI, respectively, to define significant differences between the groups. A $p$ value of $<0.05$ was considered statistically significant.

\section{Results}

Comparison of mean SBS of different pretreatment groups is shown in Table 1. The maximum SBS was seen in group III (18.84 \pm $1.04)$, followed by group II $(14.18 \pm 0.23)$, and the minimum bond strength was found in group I (13.90 \pm 1.22$)$. The ANOVA showed high statistical difference within the pretreatment groups.

Table 2 shows the comparisons between the different adhesive materials. A statistically significant difference was seen between group I vs group III and group II vs group III.

Table 3 shows the ARI scores of the different pretreatment groups. Score 0 was more in group III [8 (40\%)] followed by group II [4 (20\%)] and group I [2 (10\%)]. Least number of score 3 was found in CPP-ACP paste group. Once the ARI scores were compared within the groups, the Chi-square test showed a statistically significant probability of 0.001 .

The inference of the present study indicates that the CPP-ACP paste pretreatment improves the SBS of orthodontic bracket significantly followed by fluoride varnish and acid etching. 


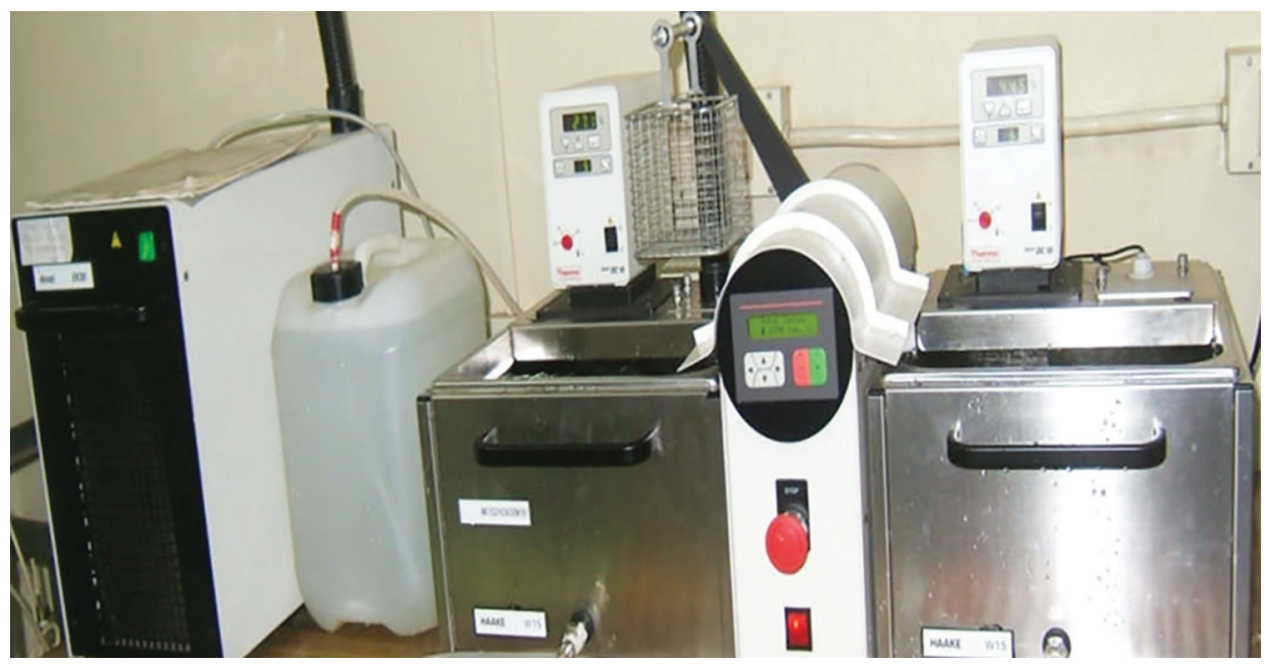

Fig. 1: Thermocycler used in the present study

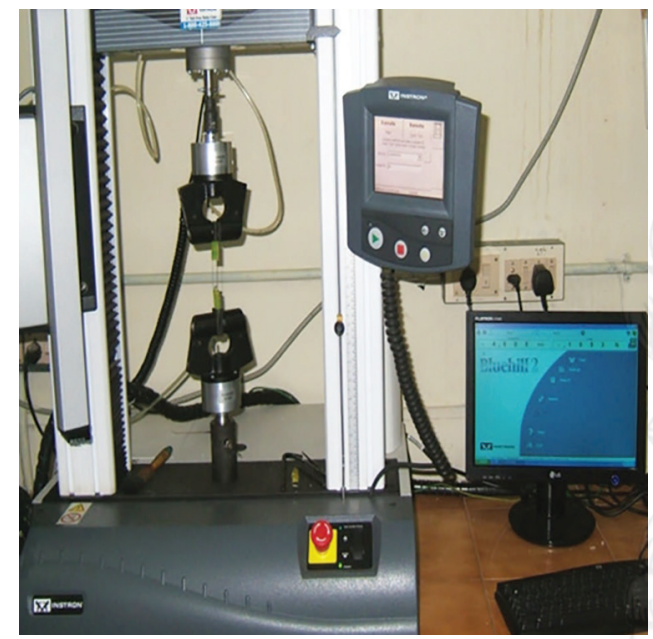

Fig. 2: Evaluation of shear bond strength using INSTRON universal testing machine

\section{Discussion}

Since the last 20 years, the research related to the strength of the bond between tooth enamel and orthodontic brackets has been remarkable. Nevertheless, there are certain unresolved issues pertaining to the selection of adhesive material by the orthodontist, owing particularly to the availability of various adhesive materials in the market. Some of the main concerns are efficiency of the adhesive agent, safety of the technique, and cost-effectiveness. Deposition of plaque around the orthodontic appliances is a prime concern as plaque can initiate dental caries and periodontal diseases. ${ }^{7}$

Numerous preventive procedures, for example, use of adhesive agents that release fluoride and the use of protective agents on the surface of enamel prior to bonding of the brackets have been proposed to avoid the above-stated problem. ${ }^{8}$ Etching of the enamel with acid causes removal of minerals from the hydroxyapatite crystals of enamel rods and uncovers the micropores on the enamel, thus permitting the interlocking of adhesive material and tooth enamel. Therefore, the orthodontic brackets could withstand the forces of mastication. Topical application of fluoride and (CPP-ACP) is permit-free ions of fluoride, calcium, and phosphate to go into the tooth enamel and to transform into tougher crystals which consecutively help in avoiding demineralization and improve remineralization. ${ }^{9}$ This process may affect the acid-etching procedure.

According to Serra and Cury, ${ }^{10}$ the integration of F released from the adhesive material with tooth enamel lowers the solubility of enamel in acidic environments. This mechanism is centered on the ability of $\mathrm{F}$ to integrate itself into the hydroxyapatite crystalline structure of the enamel and dentin leading to the formation of a mineral phase which is not soluble and resistant to carious process.

We used Transbond XT as an adhesive material. Our results showed highest SBS $(18.84 \pm 1.04)$ with Transbond XT pretreated with CPP-ACP. Likewise, Rix et al. ${ }^{11}$ found a $20.19 \mathrm{MPa}$ bond strength for Transbond XT in dry state which is significantly higher than the current study value $(10.23 \pm 2.15 \mathrm{MPa})$. The chosen teeth were exposed to a thermocycling technique in two streams of water whose temperatures were controlled and maintained at $10-55^{\circ} \mathrm{C}$ for 1 day post the 30-day incubation period. As per Bishara et al., ${ }^{12}$ "the aim of thermocycling was to expose the teeth to the changes in the temperature in the same way as it occurs in the mouth." Even they found a high bond strength of $10.99 \pm 3.34 \mathrm{MPa}$ with assure hydrophilic primer in moist conditions using human saliva. When compared to our study, this value for hydrophilic primer SBS is considerably high.

In the present study, the SBS was more in pretreated with CPP-ACP as compared with the acid-etching technique. Both Azarpazhooh and Limeback ${ }^{13}$ and Rehder Neto et al. ${ }^{14}$ mention that pretreatment of enamel with CPP-ACP that contains fluoride (900 ppm) did not compromise the bond strength of the tested self-etch and etch-and-rinse adhesive systems. Two reasons might be responsible for these findings: firstly, the sodium fluoride present in the fluoride-containing CPP-ACP paste could interact with the ACP component of the casein complex, rendering both inorganic components ineffective. Indisputably, the investigational nature of this study restricts the confirmation of this hypothesis, which requires further exploration. Secondly, the fluoride contained in the CPP-ACP compound must have been essentially laid on the surface of the enamel as nanocomplexes but then such pretreatment of undamaged enamel surface with fluoride may have mild or no adverse effect on the tested adhesives' SBS. As a result, enamel 

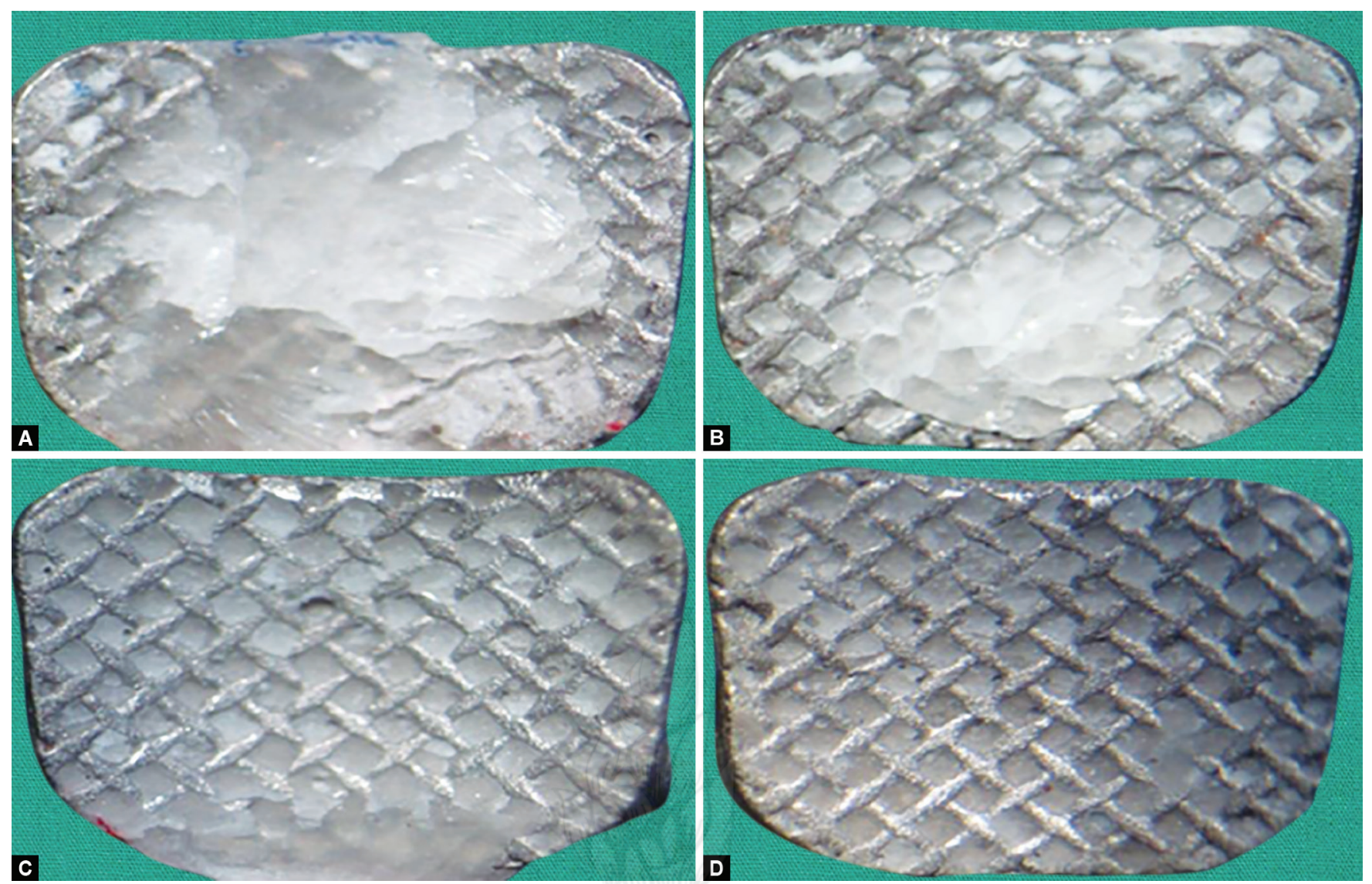

Figs 3A to D: Adhesive remnant index score: (A) Score 0; (B) Score 1; (C) Score 2; (D) Score 3

Table 1: Comparison of mean shear bond strength of different pretreatment groups

\begin{tabular}{lllll}
\hline Groups & $n$ & $\begin{array}{l}\text { Mean } \pm \text { standard } \\
\text { deviation }\end{array}$ & Fvalue & pvalue \\
\hline Group I: acid etching & 20 & $13.90 \pm 1.22$ & 24.134 & 0.0001 \\
Group II: Bifluorid 12 & 20 & $14.18 \pm 0.23$ & & HS \\
Group III: CPP-ACP paste & 20 & $18.84 \pm 1.04$ & & \\
\hline
\end{tabular}

$p<0.05$; HS, highly significant

Table 2: Multiple comparisons of different pretreatment groups using Tukey's post hoc test

\begin{tabular}{llcl}
\hline Group & Compared with & Mean difference & Significance \\
\hline Group I & Group II & -0.28 & 0.08 \\
& Group III & -4.94 & 0.001 \\
Group II & Group I & 0.28 & 0.08 \\
& Group III & -4.66 & 0.001 \\
\multirow{2}{*}{ Group III } & Group I & 4.94 & 0.001 \\
& Group II & 4.66 & 0.001 \\
\hline
\end{tabular}

pretreatment with the tried CPP-ACP complex that contains fluoride seems to be a harmless preventive (or therapeutic) technique in relation to bond strength of the brackets gotten with the existing adhesive systems.

In our study, the use of CPP-ACP and light-cure adhesives demonstrated comparatively significant superior results than the control group. Our results were similar to the results obtained by Kecik et al. ${ }^{15}$ and Xiaojun et al. ${ }^{16}$ who showed significant increase in the bond strength with topical application of CPP-ACP. However, this finding was unlike the results obtained by Tabrizi and Cakirer ${ }^{17}$ who presented rise in mean SBS but which was non-significant. Also, the combination of CPP-ACP and chemical-cure adhesive systems showed significant decline in the mean SBS compared to that of the control group.

The present study showed better bonding strength with pretreatment of Bifluorid 12 varnish than acid etching. Veli et al. ${ }^{18}$ conducted a trial on 140 newly extracted premolars that were randomized to seven groups. The control for other groups was group I. All the left-over groups were exposed to demineralization. The brackets were bonded directly to the demineralized surface of enamel in group II. Casein phosphopeptide-amorphous calcium phosphate paste, microabrasion with a mixture prepared with $18 \%$ hydrochloric acid and fine pumice powder, fluoride varnish (Bifluorid 12), resin infiltrant (Icon ${ }^{\circledR}$ ), and microabrasion with an 
Table 3: Assessment of ARI scores of different pretreatment groups

\begin{tabular}{llllll}
\hline Groups & $n$ & Score 0 (\%) & Score 1 (\%) & Score 2(\%) & Score 3 (\%) \\
\hline Group I: acid etching & 20 & $2(10)$ & $6(30)$ & $7(35)$ & $5(25)$ \\
Group II: Bifluorid 12 & 20 & $4(20)$ & $7(35)$ & $7(25)$ & $4(20)$ \\
Group III: CPP-ACP paste & 20 & $8(40)$ & $3(15)$ & $2(10)$ \\
\hline
\end{tabular}

Chi-square $\left(\chi^{2}\right)=18.82 ; p=0.001$

agent (Opalustre) were applied. Finally, they established that all the demineralization methods increase bonding to demineralized surface of enamel.

It is impossible to replicate all the factors of the oral environment in in vitro studies and this is the drawback of our study. This warrants in vivo trials in the future so as to confirm in vitro results and to establish clinically significant values of bonding between various adhesive systems.

\section{Conclusion}

This study found a significant improvement in the SBS of orthodontic brackets when pretreated with CPP-ACP paste, followed by use of fluoride varnish and acid etching. The role of fluoride in improving the crystal growth has been indicated by ARI scores. The pretreatment with the fluoride-rich materials increases the surface area for etching.

\section{References}

1. Al-Kawari HM, Al-Jobair AM. Effect of different preventive agents on bracket shear bond strength: in vitro study. BMC Oral Health 2014;14:28. DOI: 10.1186/1472-6831-14-28.

2. Fontana M, Young DA, Wolff MS, et al. Defining dental caries for 2010 and beyond. Dent Clin North Am 2010;54(3):423-440. DOI: 10.1016/ j.cden.2010.03.007.

3. da Silva SR, da Silva LAH, Basting RT, et al. Evaluation of the anticariogenic potential and bond strength to enamel of different fluoridated materials used for bracket bonding. Rev Odontol UNESP 2017;46(3):138-146. DOI: 10.1590/1807-2577.06716.

4. Margolis HC, Moreno EC. Physicochemical perspectives on the cariostatic mechanisms of systemic and topical fluorides. J Dent Res 1990;69:606-613. DOI: 10.1177/00220345900690\$119.

5. Toledano M, Osorio R, Osorio E, et al. Bond strength of orthodontic brackets using different light and self-curing cements. Angle Orthod 2003;73(1):56-63.

6. Artun J, Bergland S. Clinical trials with crystal growth conditioning as an alternative to acid-etch enamel pretreatment. Am J Orthod 1984;85(4):333-340. DOI: 10.1016/0002-9416(84)90190-8.
7. Abu Alhaija ESJ, Al-Wahadni AMS. Evaluation of shear bond strength with different enamel pre-treatments. Eur J Orthod 2004;26(2): 179-184. DOI: 10.1093/ejo/26.2.179.

8. Pseiner BC, Freudenthaler J, Jonke E, et al. Shear bond strength of fluoride-releasing orthodontic bonding and composite materials. Eur J Orthod 2010;32(3):268-273. DOI: 10.1093/ejo/cjp116.

9. Hamba H, Nikaido T, Inoue $\mathrm{G}$, et al. Effects of CPP-ACP with sodium fluoride on inhibition of bovine enamel demineralization: a quantitative assessment using micro-computed tomography. J Dent 2011;39(6):405-413. DOI: 10.1016/j.jdent.2011.03.005.

10. Serra MC, Cury JA. The in vitro effect of glass-ionomer cement restoration on enamel subjected to a demineralization and remineralization model. Quintessence Int 1992;23(2):143-147.

11. Rix D, Foley TF, Mammandras A. Comparison of bond strength of three adhesives: composite resin, hybrid gic, an glass filled gic. Am J Orthod Dentofacial Orthop 2001;119(1):36-42. DOI: 10.1067/mod.2001.110519.

12. Bishara SE, Ajlouni R, Laffoon JF. Effect of thermocycling on the shear bond strength of a cyanoacrylate orthodontic adhesive. Am J Orthod Dentofacial Orthop 2003;123(1):21-24. DOI: 10.1067/mod.2003.1.

13. Azarpazhooh A, Limeback H. Clinical efficacy of casein derivatives: a systematic review of the literature. J Am Dent Assoc 2008;139(7): 915-924. DOI: 10.14219/jada.archive.2008.0278.

14. Rehder Neto FC, Maeda FA, Turssi CP, et al. Potential agents to control enamel caries-like lesions. J Dent 2009;37(10):786-790. DOI: 10.1016/ j.jdent.2009.06.008.

15. Kecik D, Cehreli SB, Sar C, et al. Effect of acidulated phosphate fluoride and casein phosphopeptide-amorphous calcium phosphate application on shear bond strengths of orthodontic brackets. Angle Orthod. 2008;78(1):129-133. DOI: 10.2319/122506-529.1.

16. Xiaojun D, Xuehua G, Jing L, et al. Effects of CPP-ACP paste on the shear bond strength of orthodontic brackets. Angle Orthod 2009;79(5):945-950. DOI: 10.2319/101108-573.1.

17. Tabrizi A, Cakirer B. A comparative evaluation of casein phosphopeptideamorphous calcium phosphate and fluoride on the shear bond strength of orthodontic brackets. Eur J Orthod 2011;33(3): 282-287. DOI: 10.1093/ejo/cjq062.

18. Veli I, Akin M, Baka ZM, et al. Effects of different pre-treatment methods on the shear bond strength of orthodontic brackets to demineralized enamel. Acta Odontol Scand 2016;74(1):7-13. DOI: 10.3109/00016357.2014.982703. 